\title{
CTLA-4 antibodies in cancer immunotherapy
}

\begin{abstract}
Activating human immune system to battle cancer has been a focus of cancer immunotherapy research from quite some time. After decades of disappointment, the tide has finally changed with some recent successes in clinical trials. The first such approach with clinical success is cytotoxic T lymphocyte antigen-4 (CTLA-4) antibody therapy which aims to relieve the immune suppressive effects of CTLA-4 on T cells which are specifically aimed to fight cancer. CTLA-4 antibody therapy has enhanced the survival of patients suffering from late stage refractory metastatic melanoma. The success of CTLA- 4 antibody therapy has cemented cancer immunotherapy potential in patients and even swayed away skeptics.
\end{abstract}

Keywords: cancer immunotherapy, CTLA-4, anti-CTLA-4, immunosuppression, check point blockade
Volume 3 Issue 3 - 2016

\author{
Veerendra Koppolu, 'Veneela KR Vasigala ${ }^{2}$ \\ 'Department of Molecular Biosciences, University of Kansas, \\ USA \\ ${ }^{2}$ Rangaraya Medical College, NTR University of Health Sciences, \\ India
}

Correspondence: Veerendra Koppolu, Department of Molecular Biosciences, University of Kansas, Lawrence USA, Tel I 6786979216,Email veeru.bios@gmail.com

Received: June 06, 2016 | Published: June 23, 2016
Abbreviations: CTLA-4, cytotoxic T lymphocyte antigen-4; TCR, T cell receptor; TME, tumor microenvironment

\section{Introduction}

Cancer immunotherapy is a way of treating cancer by harnessing the immune system potential to fight cancer. Recent advances in understanding the biology of tumor and immune system has identified checkpoints that modulate the immune system from presenting a strong anti-tumor immune response. Naturally, these checkpoints are considered as attractive alternate targets to fight cancer in addition to the current approaches such as chemotherapy, radiotherapy, and surgery. Checkpoint blockade therapies potentially differ from other treatment approaches as they do not directly attack tumor cells but rather produce tumor specific immune response by relieving the immune system inhibition. One such approach is targeting an immune modulator cytotoxic T lymphocyte antigen (CTLA-4) expressed on activated $\mathrm{T}$ cells. Antibodies that target CTLA-4 have received clinical validation and now emerged as major advancements in cancer immunotherapy.

\section{Immune modulation by CTLA-4}

CTLA-4 is an immunosupressor involved in regulatory feedback inhibition of activated $\mathrm{T}$ cells through inhibition of CD28 dependent T cell co-stimulation. ${ }^{1}$ In a typical cancer-immunity cycle, the cancer antigens during the process of oncongenesis are presented by antigen presenting cells (dendritic cells) on their surface MHC receptors. MHC receptors presenting antigens interact with $\mathrm{T}$ cell receptor (TCR) on the surface of T cells initiating a first/primary signal of the $\mathrm{T}$ cell activation. ${ }^{2}$ Activation of $\mathrm{T}$ cells also require a secondary (costimulatory) signal facilitated through interaction of CD28, a master T cell co-stimulator expressed on T cells, and CD80 (B7.1) or CD 86 (B7.2) expressed on antigen presenting cells. ${ }^{3}$ When TCR is already engaged with $\mathrm{MHC}$ presenting antigen, CD28 engagement with B7.1/ B7.2 further amplifies the TCR signaling leading to T cell proliferation and differentiation into tumor antigen specific CD4+ helper $\mathrm{T}$ cells or $\mathrm{CD} 8+$ cytotoxic $\mathrm{T}$ cells..$^{3-6}$ The regulation of $\mathrm{T}$ cell response is a complex process where activation event can be countered by tumor cell initiated inhibition events to limit the anti-tumor immune response. CTLA-4 is one such inhibitory receptor expressed on the activated $\mathrm{T}$ cells and interacts with higher affinity to B7.1 \& B7.2 than CD28, and thus out competes CD28 binding leading to suppression of anti-tumor response. ${ }^{7,8}$
The pathway through which CTLA-4 inhibits T cell activation is under active research. Trivol, et al. ${ }^{9}$ and Waterhouse, et al. ${ }^{10}$ found that CTLA-4 gene knockout mice presents a lethal hyper immunity response indicating that CTLA-4 activity is required for inhibition of immune response (Immune tolerance). ${ }^{9,10}$ Rudd et al in 2009 showed that CTLA-4 dependent inhibition of T cell activity requires activation of phosphatase such as SHP2 and PP2A, whose expression counters the kinases signaling induced by $\mathrm{CD} 28$ and TCR. ${ }^{11}$ In addition, CTLA-4 expression interferes with the TCR initiated synapse that is required to maintain continuous interactions between TCR and MHC presented antigens. ${ }^{12}$ CTLA-4 dependent inhibition is initiated mainly in differentiated $\mathrm{T}$ cells and its expression is absent in naive $\mathrm{T}$ cells and memory T cells where CD28 is predominantly expressed. CTLA- 4 expression is quick and happens generally within one hour of antigen engagement with TCR. CTLA-4 suppressive effects are initiated primarily in secondary lymphoid organs where $\mathrm{T}$ cell activation occur rather than in the tumor microenvironment (TME) because of the limited B7.1 and B7.2 expression on non-hematological tumor cells. However, recent evidences suggest anti-tumor effects of CTLA-4 inhibition happens even when T lymphocytes movement to TME from secondary lymphoid organs is blocked, indicating at least some direct CTLA-4 role in tumor micro environment. In addition, regulatory $\mathrm{T}$ cells (Treg cells) present in tumor micro environment to counteract the $\mathrm{T}$ cell function also express CTLA-4 and thus present another evidence of direct role of CTLA-4 in tumor microenvironment.

\section{Inhibition of CTLA-4 activity as a cancer treatment strategy}

Our conventional wisdom underlying the inhibition of CTLA-4 is to release the feedback inhibition of antigen encountered T cells. James Allison group in 1996 has first ever attempted work in this direction. They found tumor rejection, including the pre-established tumors in multiple tumor murine models when CTLA- 4 activity is blocked by CTLA-4 antibody. In addition, antibodies also provided immunity to secondary exposure of animals to tumor cells. ${ }^{13-18}$ This discovery garnered further interest in testing the approach in clinical trials and ultimately led to the development of Ipilimumab, a fully human IgG1 anti-CTLA-4 antibody used for clinical testing.

Clinical trials to test this approach were attempted based on the results in the preclinical studies and the success of these trials ultimately led to the approval of Ipilimumab by FDA in 2011. Ipilimumab was tested in clinical trials for patients suffering with 
late stage metastatic melanoma who failed to respond to the standard therapies. Phase II data for these patients showed an improved clinical activity with long tern survival benefit. ${ }^{19,20}$ Based on positive phase II data, a length Phase III clinical trial was initiated on patients suffering from relapsed metastatic melanoma. Ipilimumab was given to patients either as monotherapy or in combination with glycoprotein 100 (gp100) vaccines. Patients receiving Ipilimumab either alone or in combination with gp100 vaccine showed approximately two fold increase in median survival rate (10.1 months) relative to the control group that received only gp100 vaccine (median survival 6.1 month). The Ipilimumab response is found to be durable even after 2.5 years. ${ }^{21}$ In another phase III clinical trial, patients were given a standard decarbazine therapy and later treated with Ipilimumab. ${ }^{22}$ Patients treated with Ipilimumab showed a median survival rate of 11.2 months relative to 9.1 months observed for patients that received only decarbazine care. In addition, patients that received Ipilimumab along with standard therapy showed a $21 \%$ survival after 3 years which was higher compared to $12 \%$ survival with standard therapy alone..$^{22}$ The improved clinical activity was found to be associated with increased tumor antigen specific $\mathrm{T}$ cells, increased pretreatment levels of tumor infiltrating lymphocytes and increased mutation load. Ipilimumab represented the first ever phase III clinical success data for late stage metastatic melanoma and is now a popular treatment in advancing patients survival.

The improved clinical activity of Ipilimumab is associated with some side effects such as serious grade ( 3 or 4 ) colitis and dermatitis. ${ }^{23}$ This toxicity may be expected because inhibiting this immune suppressor over activates the $T$ cells leading to inflammatory pathology. Observation of the inflammatory effect at distant sites from tumor microenvironment suggests the role of CTLA-4 in peripheral tolerance. Another limitation of this therapy is that it takes long time to build an anti-tumor response as it focuses on improving the immune response rather than directly targeting the tumor cells. During the wait time between Ipilimumab treatment and immunity build up, there is a great chance that tumors might increase in their size. It was shown that almost $10 \%$ of the patients have increased disease progression after the treatment, although disease stabilization and prolonged survival was achieved later. ${ }^{24}$ Ipilimumab is also associated with toxic effects on endocrine glands. The most notable endocrine toxicity is hypophysitis (pituitary dysfunction) and is found to occur to a variable degree $(0-25 \%)$ in metastatic melanoma patients after 2-4 months of treatment. Secondary to hypophysitis, other endocrinal side effect such as hypothyroidism and adrenal insufficiency may also occur. Corticosteroids and hormonal replacement are the current treatment choices for these patients when the above mentioned endocrine dysfunctions are diagnosed. ${ }^{25,26}$ Despite these limitations Ipilimumab provides a realistic hope for patients suffering from late stage metastatic melanoma who failed to respond to other treatment strategies.

In a broader way, anti-CTLA-4 therapy has provided a clinical validation to the cancer immunotherapy. The approval of Ipilimumab has generated enough curiosity for researchers to work on other potential immune suppressive receptors. One such receptor is PD-1 which inhibits $T$ cell activity directly in tumor micro environment. ${ }^{1,27-29}$ Recently drugs targeting PD-1 such as pembrolizumab (against metastatic melanoma) and nivolumab (against metastatic melanoma, renal cancer, and non-small cell lung cancer) gained FDA approval taking another step forward in treating patients with immunotherapy approach. ${ }^{30,31}$ Many clinical trials are also in progress to test if the anti CTLA-4 antibody therapy either alone or in combination with
PD-1 antibodies holds promise for other types of cancers such as prostate and renal cancers. Also, researchers are testing the hypothesis of further extending the survival rates by combining the immune modulating anti-CTLA-4 therapy with other treatments that directly target tumor growth and angiogenesis (targeted therapies). ${ }^{32}$

In addition to IgG1 type of anti-CTLA4 antibody (ipilimumab), clinical trials are also attempted with IgG2 isotype of CTLA4 antibody, tremelimumab, for metastatic melanoma. Although phase I and II trials with tremelimumab for metastatic melanoma showed some response, phase III trials did not show any improved clinical anti-tumor activity which lead to subsequent termination of trials. ${ }^{33}$ Researchers are still investigating tremelimumab either as monotherapy against metastatic mesothelioma or in combination with other immunotherapeutic drugs for multiple cancers, including nonsmall cell lung cancer, squamous cell carcinoma of the head and neck, bladder, pancreatic, gastric and liver cancers. Recently, tremelimumab monotherapy phase IIb trial results for metaststic melanomas were published where the therapy did not demonstrate survival benefit. ${ }^{34}$ However, the same group also published a positive clinical outcome for tremelimumab in combination with durvalumab (PD-1 antibody) for non-small cell lung cancer in a phase Ib study. ${ }^{35}$

\section{Conclusion}

Immune checkpoint therapy such as anti-CTLA-4 therapy has provided cancer patients with another treatment approach in addition to the conventional chemotherapy, radiotherapy, and surgery. The major advantages of this therapy are specificity, adaptability, and memory response to subsequent exposures leading to efficient and durable responses to treat cancer. Despite these advantages, tumor immunotherapies don't help everyone, and researchers are actively seeking the reason for it. However, for physicians accustomed to lose patients with advanced melanoma, this has become a success story and has opened a new door in the treatment.

\section{Acknowledgments}

None.

\section{Conflicts of Interset}

None.

\section{References}

1. Topalian SL, Drake CG, Pardoll DM. Immune checkpoint blockade: a common denominator approach to cancer therapy. Cancer Cell. 2015;27(4):450-461.

2. Chen DS, Mellman I. Oncology meets immunology: the cancerimmunity cycle. Immunity. 2013;39(1):1-10.

3. Linsley PS, Brady W, Grosmaire L, et al. Binding of the B cell activation antigen B7 to CD28 co stimulates T cell proliferation and interleukin 2 mRNA accumulation. J Exp Med. 1991;173(3):721-730.

4. Slaney CY, Kershaw MH, Darcy PK. Trafficking of T cells into tumors. Cancer Res. 2014;74(24):7168-7174.

5. Muller WA. Localized signals that regulate transendothelial migration. Curr Opin Immunol. 2016;38:24-29.

6. Chen L, Flies DB. Molecular mechanisms of T cell co-stimulation and co-inhibition. Nat Rev Immunol. 2017;13(4):227-242.

7. Krummel MF, Allison JP. CD28 and CTLA-4 have opposing effects on the response of T cells to stimulation. J Exp Med. 1995;182(2):459-465. 
8. Brunet JF, Denizot F, Luciani MF, et al. A new member of the immunoglobulin super family--CTLA-4. Nature. 1987;328(6127):267270 .

9. Tivol EA, Borriello F, Schweitzer AN, et al. Loss of CTLA-4 leads to massive lymph proliferation and fatal multiorgan tissue destruction, revealing a critical negative regulatory role of CTLA-4. Immunity. 1985;3(5):541-547.

10. Waterhouse P, Penninger JM, Timms E, et al. Lymph proliferative disorders with early lethality in mice deficient in Ctla-4. Science. 1995;270(5238):985-988

11. Rudd CE, Taylor A, Schneider H. CD28 and CTLA-4 coreceptor expression and signal transduction. Immunol Rev. 2009;229(1):12-26.

12. Schneider H, Downey J, Smith A, et al. Reversal of the TCR stop signal by CTLA-4. Science. 2006;313(5795):1972-1975.

13. Leach DR, Krummel MF, Allison JP. Enhancement of antitumor immunity by CTLA-4 blockade. Science. 1996;271(5256):1734-1736.

14. Hurwitz AA, Yu TF, Leach DR, et al. CTLA-4 blockade synergizes with tumor-derived granulocyte-macrophage colony-stimulating factor for treatment of an experimental mammary carcinoma. Proc Natl Acad Sci US A. 1986;95(17):10067-10071.

15. van Elsas A, Hurwitz AA, Allison JP. Combination immunotherapy of B16 melanoma using anti-cytotoxic $\mathrm{T}$ lymphocyte-associated antigen 4 (CTLA-4) and granulocyte/macrophage colony-stimulating factor (GM-CSF)-producing vaccines induces rejection of subcutaneous and metastatic tumors accompanied by autoimmune depigmentation. $J$ Exp Med. 1999;190(3):355-366.

16. Waitz R, Fasso M, Allison JP. CTLA-4 blockade synergizes with cryoablation to mediate tumor rejection. Oncoimmunology. 2012;1(4):544-546.

17. Zamarin D, Holmgaard RB, Subudhi SK, et al. Localized oncolytic virotherapy overcomes systemic tumor resistance to immune checkpoint blockade immunotherapy. Sci Transl Med. 2015;6(226):226-232.

18. Koppolu V, Osaka I, Skredenske JM, et al. Small-molecule inhibitor of the Shigella flexneri master virulence regulator VirF. Infect Immun. 2013;81(11):4220-4231.

19. O’Day SJ, Maio M, Chiarion-Sileni V, et al. Efficacy and safety of ipilimumab monotherapy in patients with pretreated advanced melanoma: a multicenter single-arm phase II study. Ann Oncol. 2010;21(8):1712-1717.

20. Camacho LH, Antonia S, Sosman J, et al. Phase I/II trial of tremelimumab in patients with metastatic melanoma. J Clin Oncol. 2009;27(7):10751081 .
21. Hodi FS, O’Day SJ, McDermott DF, et al. Improved survival with ipilimumab in patients with metastatic melanoma. $N$ Engl $\mathrm{J} \mathrm{Med}$. 2010;363(8):711-723.

22. Robert C, Thomas L, Bondarenko I, et al. Ipilimumab plus dacarbazine for previously untreated metastatic melanoma. $N$ Engl J Med. 2011;364:2517-2526.

23. Phan GQ, Yang JC, Sherry RM, et al. Cancer regression and autoimmunity induced by cytotoxic T lymphocyte-associated antigen 4 blockade in patients with metastatic melanoma. Proc Natl Acad Sci US A. 2003;100(14):8372-8377.

24. Wolchok JD, Hoos A, O'Day S, et al. Guidelines for the evaluation of immune therapy activity in solid tumors: immune-related response criteria. Clin Cancer Res. 2009;15(23):7412-7420.

25. Torino F, Corsello SM, Salvatori R. Endocrinological side-effects of immune checkpoint inhibitors. Curr Opin Oncol. 2016;28(4):278-287.

26. González-Rodríguez E, Rodríguez-Abreu D. Immune Checkpoint Inhibitors: Review and Management of Endocrine Adverse Events. Oncologist pii: the oncologist. 2015;0509.

27. Sharma P, Allison JP. The future of immune checkpoint therapy. Science. 2005;348(6230):56-61.

28. Dong H, Strome SE, Salomao DR, et al. Tumor-associated B7-H1 promotes T-cell apoptosis: a potential mechanism of immune evasion. Nat Med. 2002;8(8):793-800.

29. Skredenske JM, Koppolu V, Kolin A, et al. Identification of a smallmolecule inhibitor of bacterial AraC family activators. J Biomol Screen. 2013;18(5):588-598.

30. Khoja L, Butler MO, Kang SP, et al. Pembrolizumab. Journal for immunotherapy of cancer. 2015;3:36.

31. Robert C, Long GV, Brady B, et al. Nivolumab in previously untreated melanoma without BRAF mutation. $N$ Engl J Med. 2015;372:320-330.

32. Hughes PE, Caenepeel S, Wu LC. Targeted Therapy and Checkpoint Immunotherapy Combinations for the Treatment of Cancer. Trends Immunolm. 2016.

33. Ribas A, Kefford R, Marshall MA, et al. Phase III randomized clinical trial comparing tremelimumab with standard-of-care chemotherapy in patients with advanced melanoma. J Clin Oncol. 2013;31(5):616-622.

34. AZ'tremelimumab fails in mesothelioma trial. 2016.

35. Antonia S, Goldberg SB, Balmanoukian A, et al. Safety and antitumour activity of durvalumab plus tremelimumab in non-small cell lung cancer: a multicentre, phase 1b study. Lancet Oncol. 2016;17(3):299-308. 